\title{
miR-150 Down-Regulation Contributes to the Constitutive Type I Collagen Overexpression in Scleroderma Dermal Fibroblasts via the Induction of Integrin $\beta 3$
}

\author{
Noritoshi Honda, ${ }^{*}$ Masatoshi Jinnin, ${ }^{*}$ Tomomi Kira-Etoh, ${ }^{*}$ Katsunari Makino, ${ }^{*}$ Ikko Kajihara, ${ }^{*}$ Takamitsu Makino, ${ }^{*}$ \\ Satoshi Fukushima, ${ }^{*}$ Yuji Inoue, ${ }^{*}$ Yoshinobu Okamoto, ${ }^{\dagger}$ Minoru Hasegawa, ${ }^{\dagger}$ Manabu Fujimoto, ${ }^{\dagger}$ and Hironobu $\mathrm{Ihn}^{*}$
}

From the Department of Dermatology and Plastic Surgery, * Faculty of Life Sciences, Kumamoto University, Kumamoto; and the Department of Dermatology, ${ }^{\dagger}$ Kanazawa University Graduate School of Medical Science, Kanazawa, Japan

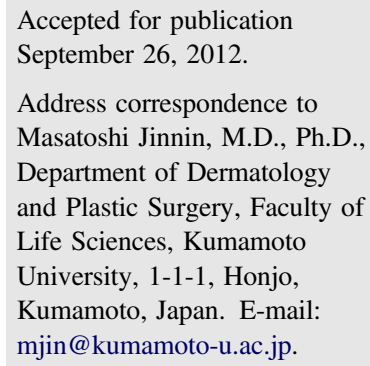

\begin{abstract}
Overexpression of integrins in dermal fibroblasts is thought to play a key role in the pathogenesis of systemic sclerosis (SSc), but the mechanism is unknown. We evaluated the possibility that microRNAs (miRNAs) are involved in the regulation of integrin $\beta 3$ in these cells. The miRNA expression profile was determined by miRNA PCR array and real-time PCR. Protein expression of integrin $\beta 3$ was determined by immunoblotting. In vivo detection of miRNA in paraffin section was performed by in situ hybridization. miR-150 expression was decreased in SSc fibroblasts both in vivo and in vitro. The transfection of miR-150 inhibitor into normal fibroblasts induced expression of integrin $\beta 3$, phosphorylated Smad3, and type I collagen, whereas forced overexpression of the miRNA resulted in their down-regulation in SSc fibroblasts. Treatment of SSc fibroblasts with 5-AdC revealed that miR-150 down-regulation in these cells is caused by DNA methylation. In addition, we found that miR-150 is detectable and quantitative in serum. Serum miR-150 levels were decreased in SSc patients, and the SSc patients with lower serum miR-150 levels tended to have more severe clinical manifestations. miR-150 may play an important role in the pathogenesis of SSc via overexpression of integrin $\beta 3$. Investigation of the regulatory mechanisms of tissue fibrosis by miR-150 could lead to development of new diagnostic tools and new treatments using miRNA. (Am J Pathol 2013, 182: 206-216; http://dx.doi.org/10.1016/ j.ajpath.2012.09.023)
\end{abstract}

Systemic sclerosis (SSc), or scleroderma, is characterized by tissue fibrosis of the skin and internal organs. Inflammation, autoimmune attack, and vascular damage are thought to trigger the activation of fibroblasts and excess production of extracellular matrix. ${ }^{1,2}$ Thus, abnormal SSc fibroblasts responsible for fibrosis may develop from a subset of cells that have escaped from normal control mechanisms. ${ }^{3,4}$

Although the mechanism of fibroblast activation in SSc skin is at present unknown, many of the characteristics of SSc fibroblasts resemble those of normal fibroblasts stimulated by transforming growth factor $\beta-1$ (TGF- $\beta 1) .{ }^{5,6}$ One of the diverse effects of TGF- $\beta 1$ on mesenchymal cells is stimulation of extracellular matrix deposition. Cultured dermal fibroblasts from affected SSc skin in vitro produce excessive amounts of various collagens, mainly type I collagen, which consists of $\alpha 1(\mathrm{I})$ and $\alpha 2(\mathrm{I})$ collagen, ${ }^{7,8}$ suggesting that the activation of SSc fibroblasts is a result of stimulation by TGF- $\beta 1$ signaling. This notion is supported by our previous findings, that i) the transcriptional activity of the $\alpha 2(\mathrm{I})$ collagen gene in SSc fibroblasts is constitutively up-regulated compared with that in normal fibroblasts but the responsiveness to ectopic TGF- $\beta 1$ is decreased in SSc cells, ${ }^{9}$ ii) Smad3, a downstream mediator of TGF- $\beta$, is constitutively phosphorylated and translocated into the nucleus in SSc fibroblasts, ${ }^{10}$ and iii) the blockade of TGF- $\beta 1$ signal using neutralizing antibody abolishes the increased expression of human $\alpha 2$ (I) collagen mRNA in SSc fibroblasts. ${ }^{11}$ On the other hand, we have previously reported that TGF- $\beta 1$ levels in the culture medium of SSc fibroblasts

Supported in part by a grant for scientific research from the Japanese Ministry of Education, Science, Sports and Culture, by Research on Intractable Diseases project funds from the Japanese Ministry of Health, Labor and Welfare, and by a Shiseido research grant. 
are not increased. ${ }^{11}$ Thus, the activation of dermal fibroblasts in SSc may result from self-activation of TGF- $\beta 1$ signaling, without any increase in TGF- $\beta 1$ concentration.

Bioactive peptide of TGF- $\beta 1$ is usually captured by latency-associated peptide- $\beta 1$ (LAP- $\beta 1$ ) noncovalently, forming the small latent complex (SLC). In this configuration, TGF- $\beta 1$ is inactive and cannot bind to its receptors. TGF- $\beta 1$ activation is a complex process involving conformational changes induced by cleavage of LAP- $\beta 1$ either by various proteases (eg, plasmin) or by physical interactions of LAP- $\beta 1$ with other proteins (eg, integrins and thrombospondin-1), leading to the release of bioactive TGF- $\beta 1 .^{12-15}$

In explanation of how TGF- $\beta 1$ is activated in SSc fibroblasts without any quantitative increase, we recently reported constitutive overexpression of integrin $\beta 3$ in these cells. The forced overexpression of integrins, including the $\beta 3$ subunit, in dermal fibroblasts results in the up-regulation of collagen expression. ${ }^{14,16-18}$ In SSc fibroblasts, the integrins may recruit and activate the SLC on the cell surface, resulting in the release of active TGF- $\beta 1$ in the pericellular region. This may enhance the incidence of interaction between active TGF- $\beta 1$ and its receptors, leading to the self-activation of SSc fibroblasts without increasing the amount of TGF- $\beta 1 .^{14,16-18}$ Integrin overexpression is therefore thought to be the most upstream event of TGF- $\beta 1$ activation and collagen upregulation in SSc fibroblasts. Nonetheless, the mechanism of integrin overexpression in SSc is still unknown.

Recently, epigenetics has attracted attention for involvement in various cellular behaviors, including cell differentiation, immune response, and organogenesis. microRNAs (miRNAs), short ribonucleic acid molecules on average only 22 nucleotides long, are post-transcriptional regulators that bind to complementary sequences in the $3^{\prime}$ untranslated regions ( $3^{\prime}$ UTRs) of mRNAs, leading to gene silencing. ${ }^{19,20}$ miRNAs have been implicated in the pathogenesis of various human diseases, including immunological disorders, cancers, and metabolic disorders. $^{21-24}$

In the present study, our objective was to evaluate the possibility that miRNAs may play some roles in the constitutive up-regulation of integrin $\beta 3$ in SSc.

\section{Materials and Methods}

\section{Cell Culture}

Human dermal fibroblasts were obtained by skin biopsy from the affected area (dorsal forearm) of five patients with diffuse cutaneous SSc and $<2$ years of skin thickening, as described previously. ${ }^{25}$ Control fibroblasts were obtained by skin biopsies from five healthy donors. Institutional review board approval and written informed consent were obtained according to the Declaration of Helsinki.

Primary explant cultures were established in $25-\mathrm{cm}^{2}$ culture flasks in modified Eagle's medium supplemented with 10\% fetal calf serum and antibiotic-antimycotic (Invitrogen; Life Technologies, Carlsbad, CA). Monolayer cultures independently isolated from different individuals were maintained at $37^{\circ} \mathrm{C}$ in $5 \% \mathrm{CO}_{2}$-enriched air. Fibroblasts between the third and sixth subpassages were used for experiments. Cells were serum-starved for 12 to 24 hours before experiments.

\section{Patient Material}

Skin samples were obtained from affected skin of five SSc patients. Control skin samples were obtained from routinely discarded skin of healthy human subjects undergoing skin graft. ${ }^{26}$

Serum samples were obtained from 40 patients with SSc; 20 cases were classified as diffuse cutaneous SSc (dcSSc) and 20 as limited cutaneous SSc (lcSSc) according to the classification system proposed by LeRoy et al. ${ }^{27}$ Control samples were collected from 20 healthy age- and sexmatched volunteers. Five patients with dermatomyositis or systemic lupus erythematosus were also included in the study as disease controls. Institutional review board approval and written informed consent were obtained according to the Declaration of Helsinki before patients and healthy volunteers were entered into the study.

\section{Mice}

Heterozygous TSK/+ mice on a C57BL/6 background were purchased from the Jackson Laboratory (Bar Harbor, ME). All mice were housed in a specific pathogen-free barrier facility. The Committee on Animal Experimentation of Kanazawa University Graduate School of Medical Science approved all studies and procedures. ${ }^{28}$

\section{In Situ Hybridization}

In situ hybridization was performed with $5^{\prime}$-locked digoxigenin-labeled nucleic acid probes complementary to human mature miR-150 and scrambled negative control (Exiqon, Vedbaek, Denmark). ${ }^{29-31}$

\section{Cell Lysis and Immunoblotting}

Fibroblasts were washed with cold PBS twice and lysed in denaturing cell extraction buffer (Biosource International, Camarillo, CA). Aliquots of cell lysates, normalized for protein concentrations, were subjected to electrophoresis as described previously. ${ }^{32}$

\section{RNA Isolation and Quantitative Real-Time PCR}

Total RNA was extracted from cultured cells using ISOGEN reagent (Nippon Gene, Tokyo, Japan). cDNA was synthesized from total RNA with a PrimeScript RT reagent kit (Takara Bio, Otsu, Japan). The primer set for GAPDH was purchased from SA Biosciences (Frederick, MD), and the primers for TGF- $\beta 1$ were from Takara Bio. DNA was 
amplified for 40 cycles of denaturation for 15 seconds at $95^{\circ} \mathrm{C}$ and annealing for 30 seconds at $65^{\circ} \mathrm{C}$.

\section{miRNA Extraction and PCR Array Analysis}

miRNA isolation from cultured cells or skin tissue was performed using an $\mathrm{RT}^{2}$ qPCR-grade miRNA isolation kit (SABiosciences, Frederick, MD) or a miRNeasy FFPE kit (Qiagen, Valencia, CA), respectively. miRNAs were reverse-transcribed into first-strand cDNA using an $\mathrm{RT}^{2}$ miRNA first-strand kit (SABiosciences). For the $\mathrm{RT}^{2}$ Profiler PCR array (SABiosciences), the cDNA was mixed with $\mathrm{RT}^{2}$ SYBR Green/ROX qPCR master mix, and the mixture was added into a 96-well $\mathrm{RT}^{2}$ miRNA PCR array (SABiosciences), which included primer pairs for 88 human miRNAs. PCR was performed on a Takara thermal cycler Dice real-time system (model TP800) according to the manufacturer's protocol, and $\mathrm{C}_{\mathrm{T}}$ values for each miRNA were extracted using thermal cycler Dice real-time system software version 2.10B (Takara Bio).

For quantitative real-time (q)PCR, primers for miR-150 or U6 (SABiosciences) and templates were mixed with SYBR Premix Ex Taq II (Takara Bio). DNA was amplified for 40 cycles of denaturation for 5 seconds at $95^{\circ} \mathrm{C}$ and annealing for 30 seconds at $60^{\circ} \mathrm{C}$. Data generated from each PCR reaction were analyzed using thermal cycler Dice realtime system software version 2.10B (Takara Bio). Transcript levels of miR-150 were normalized to U6.

\section{miRNA Extraction from Serum and PCR Analysis of miRNA Expression}

miRNA isolation from serum samples was performed with a miRNeasy RNA isolation kit (Qiagen) according to the manufacturer's instructions, with minor modification. ${ }^{33}$ Briefly, $100 \mu \mathrm{L}$ of serum was supplemented with $5 \mu \mathrm{L}$ of $5 \mathrm{fmol} / \mu \mathrm{L}$ synthetic nonhuman miRNA (Caenorhabditis elegans miR-39; Takara) as control, providing an internal reference for normalization of technical variation between samples. QIAzol lysis reagent $(1 \mathrm{~mL})$ was added and mixed well by vortexing, and then samples were incubated at room temperature for 5 minutes. Aqueous and organic phase separation was achieved by the addition of chloroform. The aqueous phase was applied to an RNeasy spin column and an RNeasy MinElute spin column (Qiagen). miRNA was eluted from the column with nuclease-free water.

cDNA was synthesized from miRNA with a Mir-X miRNA first-strand synthesis and SYBR qRT-PCR kit (Takara Bio). qPCR with a Takara thermal cycler Dice system (model TP800) used primers and templates mixed with the SYBR Premix. The sequence of the hsa-miR-150 primer $\left(5^{\prime}-\mathrm{TC}\right.$ TCCCAACCCTTGTACCAGTG-3') was designed based on the miRBase archive (http://www.mirbase.org, last accessed November 2011). ${ }^{34-37}$ DNA was amplified for 40 cycles of denaturation for 5 seconds at $95^{\circ} \mathrm{C}$ and annealing for 20 seconds at $60^{\circ} \mathrm{C}$. The relative gene expression of hsa-miR-150 and of cel-miR-39 was calculated by the standard curve method. The transcript level of miR-150 was normalized to that of cel-miR-39.

\section{Transient Transfection}

miRNA inhibitors, mimics, and miScript Target protectors were purchased from Qiagen. Lipofectamine RNAiMAX (Invitrogen; Life Technologies) was used as transfection reagent. For reverse transfection, miRNA inhibitors $(50 \mathrm{nmol} / \mathrm{L})$, mimics $(5 \mathrm{nmol} / \mathrm{L})$, and protectors $(500 \mathrm{nmol} / \mathrm{L})$ mixed with transfection reagent were added when cells were plated, followed by incubation for 96 hours at $37^{\circ} \mathrm{C}$ in $5 \%$ $\mathrm{CO}_{2}$-enriched air. Control experiments showed transcript levels for target of miRNA inhibitors to be reduced by $>80 \%$, and expression of miRNAs was increased at least fivefold by the transfection of mimics (data not shown).

\section{Luciferase Reporter Assay}

A luciferase reporter plasmid containing the integrin $\beta 3$ 3'UTR was purchased from GeneCopoeia (HmiT009818MT01; Rockville, MD). Lipofectamine 2000 (Invitrogen; Life Technologies) was used as transfection reagent. miRNA inhibitors $(50 \mathrm{nmol} / \mathrm{L})$, mimics $(5 \mathrm{nmol} / \mathrm{L})$, and reporter plasmid mixed with transfection reagent were added when cells were plated, followed by incubation for 48 hours at $37^{\circ} \mathrm{C}$ in $5 \% \mathrm{CO}_{2}$-enriched air. A Luc-Pair miR luciferase assay (GeneCopoeia) and a FilterMax F5 microplate reader (Molecular Devices, Sunnyvale, CA) were used to analyze luciferase expression according to the manufacturer protocols.

\section{Demethylation of DNA by 5-AdC}

SSc fibroblasts were treated with or without $5 \mu \mathrm{mol} / \mathrm{L} 5$-azadeoxycytidine (5-AdC; Wako Pure Chemical Industries, Osaka, Japan) for 4 days. ${ }^{38}$ Cells were subjected to miRNA expression analysis.

\section{Statistical Analysis}

Statistical analysis was performed with a Student's $t$-test, $U$-test, or Fisher's exact probability test. $P$ values of $<0.05$ were considered significant.

\section{Results}

Several miRNAs Are Down-Regulated in SSc Fibroblasts

As an initial experiment, we performed miRNA PCR array analysis with 88 miRNAs involved in human cell differentiation and development. A mixture of equal amounts of miRNAs from five normal fibroblasts and five SSc fibroblasts was prepared, and miRNA expression profile in each cell group was evaluated using PCR array. Several miRNAs 
were up-regulated or down-regulated in SSc fibroblasts, compared with normal fibroblasts (Table 1). From among these, we focused on miR-150 as the regulator of integrin $\beta 3$, according to miRNA target gene prediction. Several leading online software tools identified integrin $\beta 3$ as a putative target of miR-150, including TargetScan version 6.2 (http://www.targetscan.org, last accessed June 1, 2012), ${ }^{39-42}$ MiRanda (http://www.microrna.org, last accessed August 1,

Table 1 miRNA Expression in SSc Fibroblasts Relative to Normal Fibroblasts, by PCR Analysis

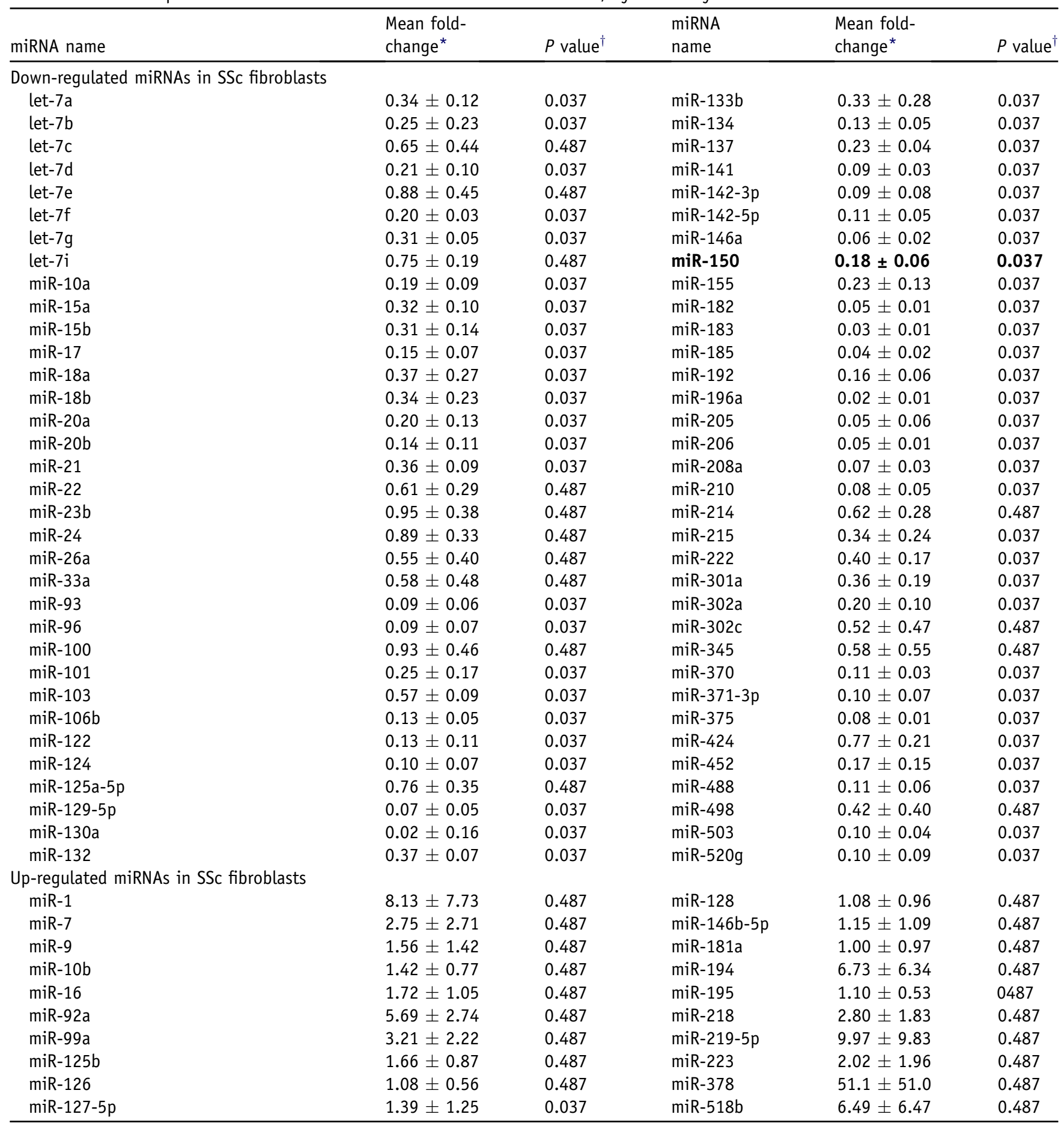

A mixture of equal amounts of miRNAs from normal $(n=5)$ or SSC $(n=5)$ fibroblasts was prepared, and the miRNA expression profile in each cell group was evaluated using PCR array. The miRNA of interest for this study (miR-150, the regulator of integrin b3) is highlighted in bold.

${ }^{*}$ Fold change was calculated as one half of the difference between the raw $C_{T}$ value of each miRNA and the $C_{T}$ value of the small RNA housekeeping gene in SSc fibroblasts, divided by one half of the difference between the raw $C_{T}$ value of each miRNA and the $C_{T}$ value of the small RNA housekeeping gene in normal fibroblasts. Data are expressed as mean fold change \pm SE of three independent experiments.

${ }^{\dagger} P$ values were calculated using the $U$-test. 
2010), ${ }^{43-46}$ DIANAmicroT version 3.0 (http://diana.cslab. ece.ntua.gr, last accessed February 1, 2009), ${ }^{4,48}$ and miRDB (http://mirdb.org/miRDB, last accessed January 1, 2012). ${ }^{49,50}$

The expression of miR-150 was down-regulated in SSc fibroblasts (0.186-fold change according to the $\Delta \Delta \mathrm{C}_{\mathrm{T}}$ method) in the array. To confirm the results obtained by miRNA PCR array, we performed qPCR analysis using a specific primer for miR-150. As expected, miR-150 was decreased in all five SSc fibroblast samples (Figure 1A), and the decrease of miR-150 in these cells was statistically significant, compared with normal cells (Figure 1B).

A

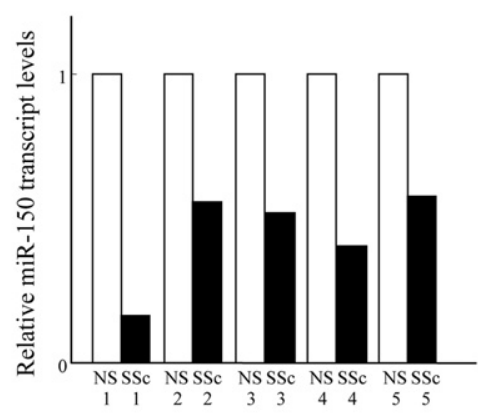

D

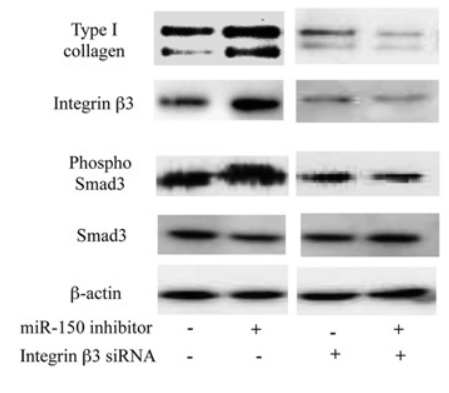

$\mathbf{E}$

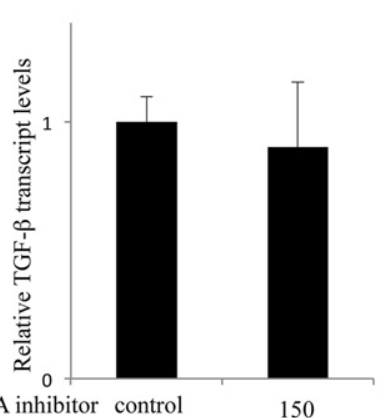

$\mathbf{F}$

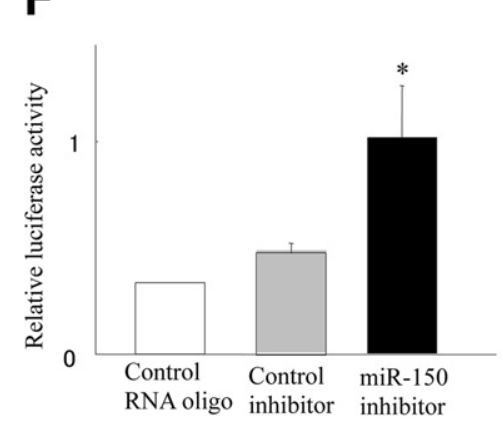

C
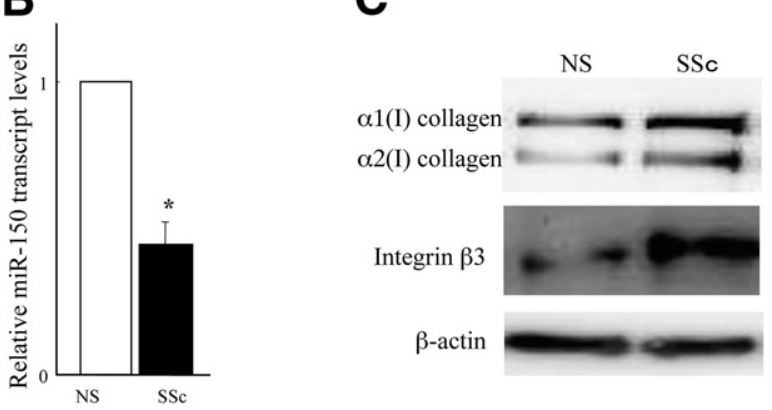

Integrin $\beta 3$

Type I collagen

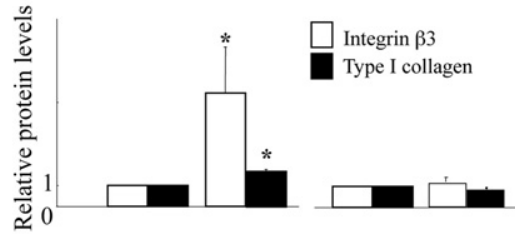

G

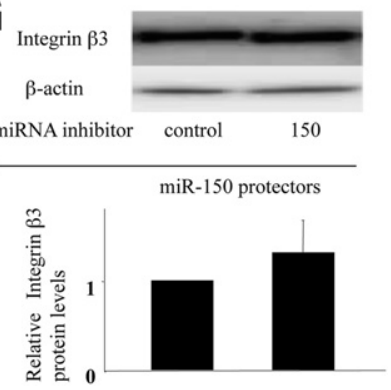

Figure 1 The effects of miR-150 inhibitor on the expression of integrin $\beta 3$ and type I collagen in normal dermal fibroblasts. A: Normal $(n=5)$ and SSc $(n=5)$ fibroblasts were serum starved for 24 hours. Total miRNA was extracted, and the relative level of miR-150 (normalized to U6) in each of the NS and SSC fibroblast samples was determined by qPCR. The values in NS fibroblasts were set at 1. B: The mean relative transcript level of miR-150 was significantly lower in SSc fibroblasts. C: NS and SSc fibroblasts were cultured independently under the same conditions until they were confluent, and then were serum starved for 24 hours. Cell lysates were subjected to immunoblotting with antibody for integrin $\beta 3$ or type I collagen. The same membrane was reprobed with anti- $\beta$-actin antibody as a loading control. Both collagen and integrin expression was increased in SSc fibroblasts. Representative results are shown. D: Normal fibroblasts at a density of $2 \times 10^{4}$ cells/well in 24-well culture plates were transfected with control miRNA inhibitor or miR-150 inhibitor in the presence of control or integrin $\beta 3$ siRNA for 96 hours. Cell lysates were subjected to immunoblotting as described for panel C. Protein levels of integrin $\beta 3$ and type I collagen quantitated by scanning densitometry and corrected for $\beta$-actin levels in the same samples are shown relative to the level in cells transfected with control inhibitor (set at 1.0). Silencing increased integrin $\beta 3$ expression and induced collagen expression. E: Normal human fibroblasts at a density of $2 \times 10^{4}$ cells/well in 24-well culture plates were transfected with control miRNA inhibitor or miR-150 inhibitor for 96 hours. Total mRNA was extracted, and the relative level of TGF- $\beta 1$ (normalized to GAPDH) was determined by qPCR as described under Materials and Methods. The values in cells with control miRNA inhibitor were set at 1 . Silencing did not affect TGF- $\beta 1$ transcript levels. F: Normal human fibroblasts at a density of $1 \times 10^{4}$ cells/well in 96-well culture plates were transfected with luciferase reporter containing the $3^{\prime}$ UTR segment of integrin $\beta 3$ and miR-150 inhibitor for 24 hours. Control miRNA inhibitor and control RNA oligo to an unrelated region of the integrin $3^{\prime}$ UTR were also included. The miR-150 inhibitor induced luciferase activity. G: Normal fibroblasts at a density of $2 \times 10^{4}$ cells/well in 24-well culture plates were transfected with control or miR-150 inhibitor in the presence of miScript Target protector specific for the miR-150 binding site on the integrin $\beta 33^{\prime}$ UTR for 96 hours. Cell lysates were subjected to immunoblotting as described for panel $C$ and the signals were quantitated as described for panel $\mathbf{D}$. Cotransfection of the protector blocked the miR-150 inhibitor-induced up-regulation of integrin $\beta 3$. Data are expressed as means \pm SE of three independent experiments. $n=5$ per group. ${ }^{*} P<0.05$ versus NS or control. 
miR-150 Regulates Type I Collagen Expression via the Direct Interaction with Integrin $\beta 3$ in Normal Fibroblasts

The expression of integrin $\beta 3$ and of both $\alpha 1$ (I) and $\alpha 2$ (I) collagen protein was increased in SSc fibroblasts (Figure 1C). Next, we determined the contribution of miR-150 to the expression of integrin $\beta 3$ and type I collagen. Silencing miR150 by the transfection of specific inhibitor for miR-150 into normal fibroblasts resulted in the increase of integrin $\beta 3$ expression (Figure 1D). Type I collagen expression was also induced by miR-150 inhibitor (Figure 1D), probably subsequent to the overexpression of integrin $\beta 3$ and phosphorylated levels of Smad3. Consistent with the hypothesis, in the presence of integrin $\beta 3$ siRNA, miR-150 inhibitor could not increase expression of phosphorylated Smad3 and type I collagen. Additionally, transfection of miR-150 inhibitor had no effect on TGF- $\beta$ transcript levels (Figure 1E). Thus, miR150 regulates TGF- $\beta$ activation via the integrin without changing TGF- $\beta$ levels directly. These results indicate that
A

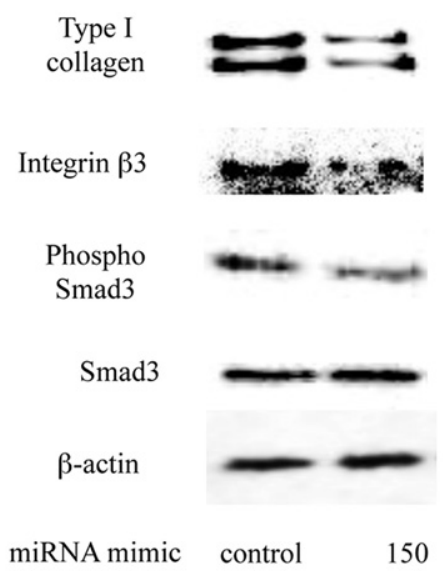

C

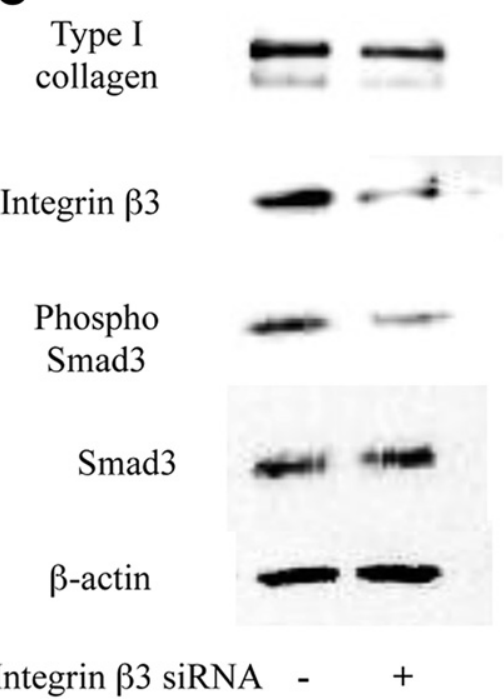

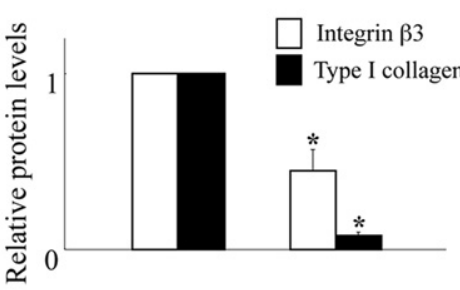

D

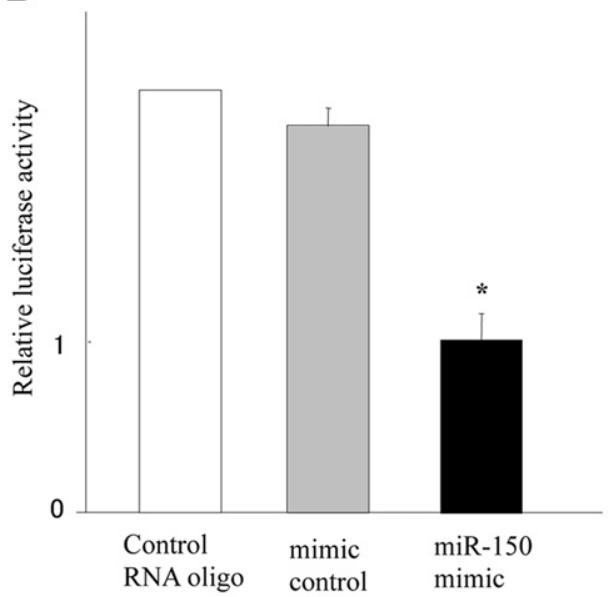

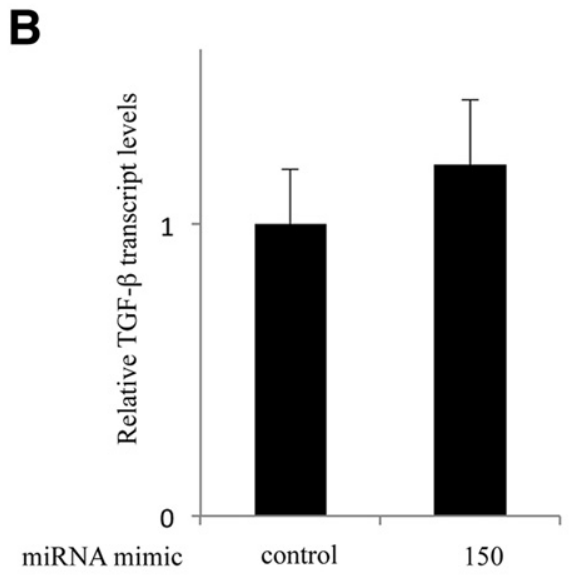

$\mathbf{E}$

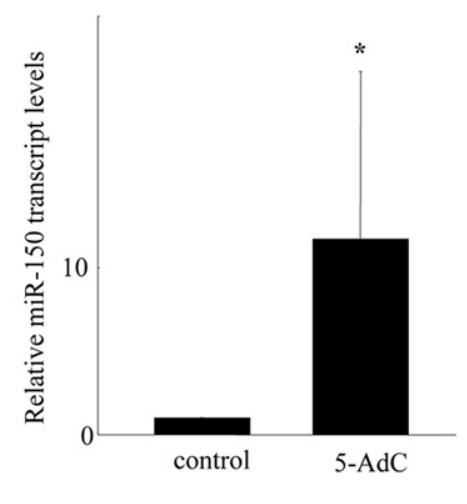

Figure 2 The effect of miR-150 mimic on the expression of integrin $\beta 3$ and type I collagen in SSc fibroblasts. A: SSc fibroblasts at a density of $2 \times 10^{4}$ cells $/$ well in 24-well culture plates were transfected with control miRNA mimic or miR-150 mimic for 96 hours, which resulted in a decrease of up-regulation of both integrin $\beta 3$ and type I collagen. Cell lysates were subjected to immunoblotting and the signals were quantitated as described for Figure 1D. B: SSc fibroblasts at a density of 2 $\times 10^{4}$ cells/well in 24-well culture plates were transfected with control miRNA mimic or miR-150 mimic for 96 hours. Total mRNA was extracted, and the relative level of TGF- $\beta$ (normalized to GAPDH) was determined by qPCR. No significant differences were revealed. The values in cells with control miRNA mimic were set at 1. C: SSc fibroblasts at a density of $2 \times 10^{4}$ cells/well in 24-well culture plates were transfected with control siRNA or integrin $\beta 3$ siRNA for 96 hours, which resulted in a decrease in up-regulation of phosphorylated Smad3 and type I collagen. Cell lysates were subjected to immunoblotting as described for Figure 1D. D: SSC fibroblasts at a density of $1 \times 10^{4}$ cells/well in 96 -well culture plates were transfected with luciferase reporter containing the $3^{\prime} U$ TR segment of integrin $\beta 3$ and miR150 mimic for 24 hours, which resulted in a decrease of luciferase activity. Control miRNA mimic and control RNA oligo to an unrelated region of the integrin $3^{\prime} U$ UTR were included. E: SSc dermal fibroblasts were cultured in six-well plates and treated with $5 \mu \mathrm{mol} / \mathrm{L} 5$-AdC, which increased expression of miR-150 (normalized to U6) more than 10-fold, as determined by real-time PCR. Data are expressed as means \pm SE of three independent experiments. ${ }^{*} P<0.05$ versus control. 
normal fibroblasts transfected with miR-150 inhibitor resemble SSc fibroblasts.

To determine whether miR-150 directly regulates the expression of integrin $\beta 3$, we performed reporter assay using luciferase construct containing the integrin $\beta 33^{\prime} \mathrm{UTR}$. miR-150 inhibitor induced the luciferase activity, in contrast to control inhibitor and control RNA oligo to an unrelated region of the integrin $\beta 33^{\prime} \mathrm{UTR}$ (Figure 1F). In addition, we used miRNA inhibitor and miScript Target protector for the miR-150 binding site on the integrin $\beta 33^{\prime}$ UTR (Qiagen), single-stranded modified RNAs designed to specifically interfere with their interaction. ${ }^{51}$ Cotransfection of the protector (Figure 1G) blocked the miR-150 inhibitorinduced up-regulation of integrin $\beta 3$ (Figure 1D), suggesting direct interaction between them.

\section{Low miR-150 Expression Leads to Up-Regulation of $\beta 3$} Integrin in SSc Fibroblasts

We then determined whether supplementation with miR-150 can normalize the SSc phenotype in SSc fibroblasts. Forced overexpression of miR-150 using miR-150 mimic decreased the up-regulated expression of integrin $\beta 3$, phosphorylated Smad3, and type I collagen in SSc fibroblasts (Figure 2A). Consistent with the data presented in Figure 1E, the transfection of miR-150 mimic into SSc fibroblasts had no effect on TGF- $\beta$ levels (Figure 2B). Transfection of integrin $\beta 3$
siRNA into SSc fibroblasts (Figure 2C) showed effects on the expression of phosphorylated Smad3 and type I collagen similar to those of miR-150 mimic (Figure 2A). These results also suggest that miR-150 plays some roles in the constitutively up-regulated collagen expression in SSc fibroblasts via the induction of integrin $\beta 3$ and the activation of TGF- $\beta$ signaling. miR-150 mimic significantly decreased the luciferase activity of integrin in SSc fibroblasts, indicating that down-regulated miR-150 in SSc fibroblasts directly up-regulates integrin $\beta 3$ (Figure 2D).

Several reports indicate that hypermethylation of miRNA loci is involved in the regulation of miRNA expression. ${ }^{52}$ We therefore determined the effect of 5-AdC, a cytosine analog refractory to methylation and used to relieve the inhibitory effects by DNA methylation in situ, ${ }^{38}$ on the down-regulated miR-150 in SSc fibroblasts. The expression of miR-150 in SSc fibroblasts treated with 5-AdC was significantly increased, compared with controls (Figure 2E). Thus, the down-regulation of miR-150 in SSc fibroblasts is likely to be regulated, at least in part, by DNA methylation.

\section{miR-150 Expression in SSc Fibroblasts in Vivo}

We then tried to determine the role of miR-150 in tissue fibrosis in vivo. Histopathologically, compared with normal tissue (Figure 3, A and B), SSc skin is characterized by dermal fibrosis due to thickened and increased collagen fibers (Figure 3, C and D). In situ hybridization showed that
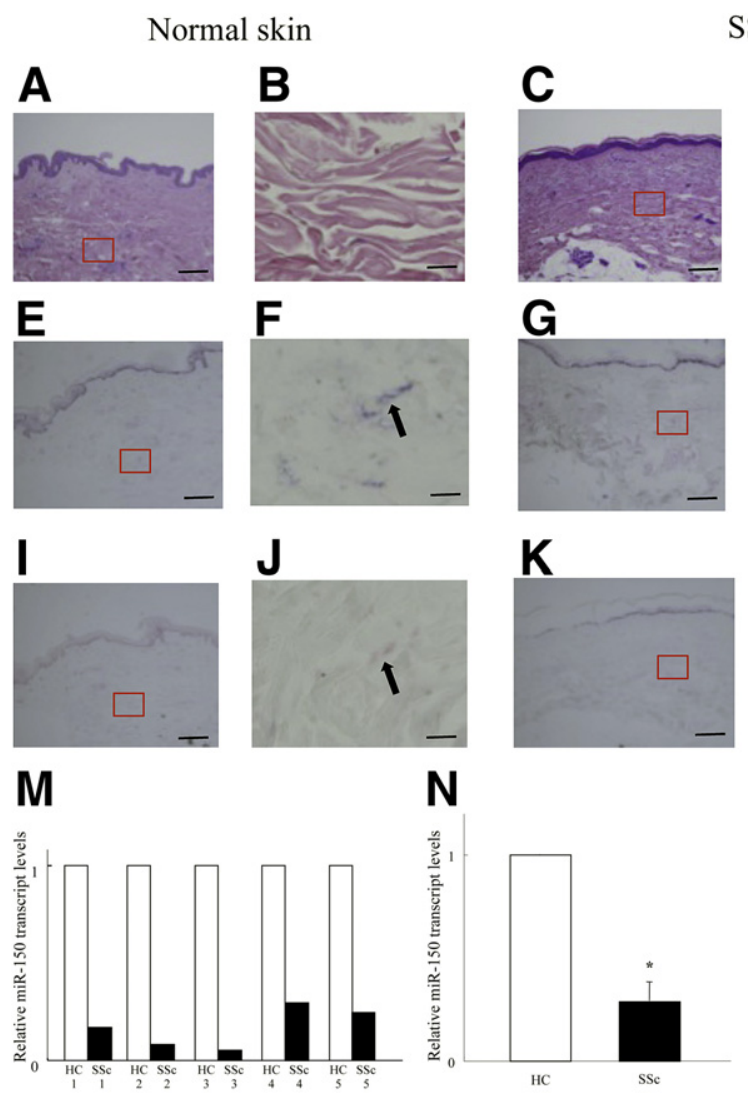

$\mathrm{SSc}$
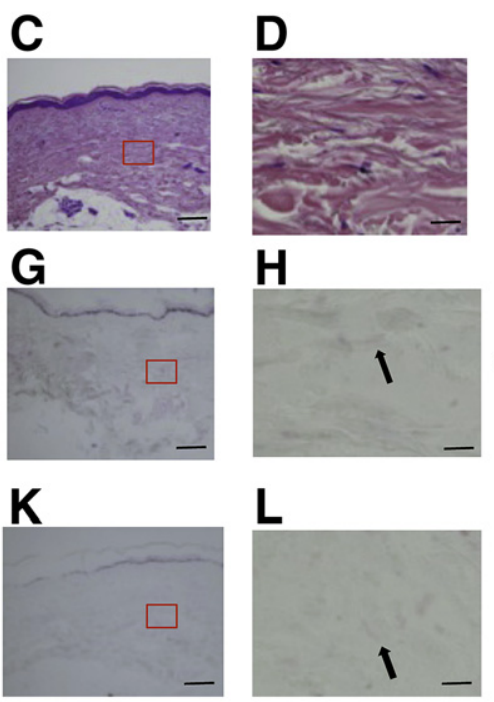

negative control
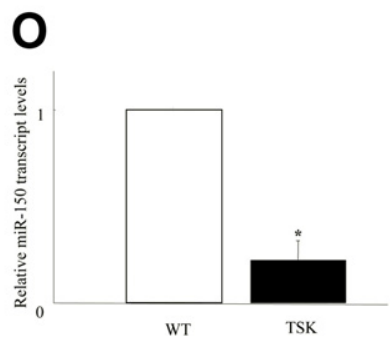

Figure 3 In vivo expression of miR-150 in SSc skin. $\mathbf{A}-\mathbf{D}$ : H\&E staining of normal (A and $\mathbf{B})$ and SSc (C and D) skin. E-L: In situ detection of miR-150 (E-H) in paraffin-embedded, formalinfixed tissues of normal (E and $\mathbf{F}$ ) and SSc ( $\mathbf{G}$ and $\mathbf{H}$ ) skin. Nuclei were counterstained with Nuclear Fast Red. The miR-150 stained blue. Dermal fibroblasts are indicated by arrows. Negative controls using scrambled probe are also shown $(\mathbf{I}-\mathbf{L})$. Boxed regions correspond to the adjacent higher-power image. $\mathbf{M}$ and $\mathbf{N}$ : Expression levels of miR-150 were decreased in five SSc skin samples, compared with five healthy control $(\mathrm{HC})$ skin samples, as determined by real-time PCR. The transcript levels in samples from HC skin were set at 1. 0: Total miRNA was extracted from skin tissue derived from five TSK mice (TSK) and three normal mice (WT). Expression levels of miR-150 were significantly lower in TSK mice, compared with WT (set at 1.0), as determined by real-time $\mathrm{PCR}$. Data are expressed as means $\pm \mathrm{SE} .{ }^{*} P<$ 0.05 versus control. Original magnification: $\times 40$

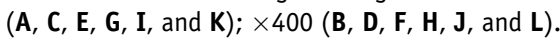
Scale bars: $200 \mu \mathrm{m}(\mathbf{A}, \mathbf{C}, \mathbf{E}, \mathbf{G}, \mathbf{I}$, and $\mathbf{K}) ; 20 \mu \mathrm{m}$ (B, D, F, H, J, and L). 

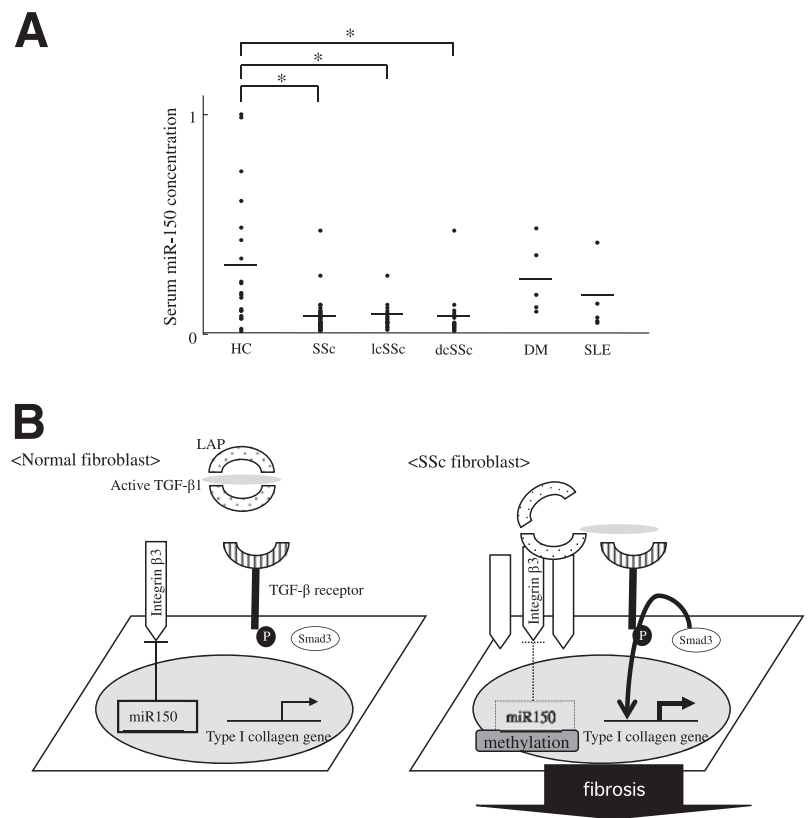

Figure 4 A: Serum miR-150 levels were lower in SSc patients. Serum miR-150 levels (normalized to cel-miR-39) were measured in 20 healthy control (HC), 20 limited cutaneous SSc (ICSSc), 20 diffuse cutaneous SSC $(\mathrm{dcSSc}), 5$ dermatomyositis (DM), and 5 systemic lupus erythematosus (SLE) samples by qPCR. The maximum value in $\mathrm{HC}$ was set at 1 . Means are indicated by horizontal bars. B: Model of the role of miR-150 in SSC fibroblasts. In SSc fibroblasts, miR-150 expression is constitutively downregulated, probably because of hypermethylation of the promoter. Decreased miR-150 causes up-regulation of $\beta 3$ integrin, which results in the activation of TGF- $\beta 1$ on the cell surface by the removal of latencyassociated peptide (LAP). The bioactive TGF- $\beta 1$ binds to its receptor and stimulates Smad3 phosphorylation/translocation into the nucleus, leading to the abnormal transcriptional activation of collagen gene in SSc fibroblasts and tissue fibrosis. ${ }^{*} P<0.05$ versus $\mathrm{HC}$.

signal for miR-150 was evident in fibroblasts of normal skin (Figure 3, E and F), but was scarcely detected in SSc fibroblasts between the thickened collagen bundles (Figure 3, G and $\mathrm{H}$ ). No signal was found in negative controls (Figure 3, I-L). The expression of miR-150 in lymphocytes, endothelial cells, and fat cells did not differ between normal skin and SSc skin (not shown). We also performed quantitative analysis of the miR-150 expression in normal and SSc skin. Compared with five normal skin samples, all five SSc skin samples showed decreased miR150 expression (Figure 2M), and the decreases of miR150 in SSc skin were statistically significant, relative to the value in normal skin (Figure $2 \mathrm{~N}$ ), which is consistent with the in vitro results (Figure 1, A and B).

To further investigate the miR-150 expression in vivo, miRNA was purified from paraffin-embedded sections derived from TSK mice, the animal fibrotic skin model. Real-time PCR revealed that mean miR-150 levels in the skin of TSK mice were significantly lower than those in wild-type mice (Figure 2O). Therefore, both in vitro and in vivo, the expression of miR-150 was likely to be decreased in fibrotic conditions.

\section{Correlation of Serum miR-150 Levels with Clinical Manifestations and Laboratory Data in SSc Patients}

Recent reports indicate that serum miRNAs can be novel biomarkers in various diseases. Serum miRNAs are thought to be stable, because they can be encapsulated in microvesicles shed from cell plasma membrane and protected from RNase. ${ }^{53-57}$ Furthermore, miRNAs in the microvesicles can be incorporated into other cells, and may alter gene expression. ${ }^{57}$ Thus, serum miRNA levels may not be merely secreted from apoptotic cells, but may exert some biological effects. We determined serum concentration of miR-150 in SSc patients and evaluated the possibility that serum miR-150 levels can be a disease marker.

There has been no previous report demonstrating the expression of miR-150 in cell-free body fluid. To validate that this miRNA is indeed detectable in human serum,

Table 2 Correlation of Serum miR-150 Levels with Clinical and Serological Features in SSc Patients

\begin{tabular}{|c|c|c|}
\hline $\begin{array}{l}\text { Clinical and serological } \\
\text { features }\end{array}$ & $\begin{array}{l}\text { Patients with } \\
\text { normal miR-150 } \\
\text { levels }(n=13)\end{array}$ & $\begin{array}{l}\text { Patients with } \\
\text { lower miR-150 } \\
\text { levels }(n=27)\end{array}$ \\
\hline Age at onset (years) & 63.0 & 58.0 \\
\hline Duration of disease (years) & 5.26 & 4.46 \\
\hline Type, diffuse:limited $(n: n)$ & $5: 8$ & $15: 12$ \\
\hline MRSS (score) & 8.3 & 14.0 \\
\hline \multicolumn{3}{|l|}{ Clinical features (\%) } \\
\hline Pitting scars or ulcers & 12.5 & $58.3^{*}$ \\
\hline Nailfold bleeding & 30.0 & 50.0 \\
\hline Raynaud's phenomenon & 80.0 & 95.6 \\
\hline Telangiectasia & 33.3 & 44.4 \\
\hline Contracture of phalanges & 87.5 & 84.6 \\
\hline Calcinosis & 0 & 0 \\
\hline Diffuse pigmentation & 33.3 & 44.4 \\
\hline Short sublingual frenulum & 87.5 & 84.6 \\
\hline Sicca symptoms & 50.0 & 71.4 \\
\hline \multicolumn{3}{|l|}{ Organ involvement (\%) } \\
\hline Pulmonary fibrosis & 45.4 & 28.0 \\
\hline Mean vital capacity & 92.0 & 94.2 \\
\hline Mean DLCO & 73.5 & 78.6 \\
\hline Pulmonary hypertension & 50.0 & 68.0 \\
\hline Esophagus & 30.0 & 17.3 \\
\hline Heart & 45.5 & 33.3 \\
\hline Kidney & 0 & 10.5 \\
\hline Joint & 66.6 & 28.6 \\
\hline Thrombosis & 0 & 0 \\
\hline \multicolumn{3}{|l|}{ ANA specificity (\%) } \\
\hline Anti-topoisomerase I & 0 & $43.4^{*}$ \\
\hline Anti-centromere & 60.0 & 47.8 \\
\hline Anti-U1 RNP & 10.0 & 17.4 \\
\hline
\end{tabular}

The cutoff value was set at the mean value of the patients.

${ }^{\star} P<0.05$ versus patients with normal serum miR-150 levels; Fisher's exact probability test or $U$-test.

ANA, antinuclear antibodies; DLCO, diffusion capacity of the lung for carbon monoxide; MRSS, Modified Rodnan Skin Score; RNP, ribonucleoprotein. 
miRNA was extracted from sera of healthy individuals and the level of miR-150 was determined by qPCR using a primer set specific for miR-150 (Supplemental Figure S1). The amplification of miR-150 was observed, and $C_{T}$ values were increased by the serial dilution of the miRNA. Thus, miR-150 was shown to be detectable and quantifiable in the serum using our method.

Serum samples were obtained from 40 patients ( 9 men, 31 women) with SSc (20 dcSSc, $20 \mathrm{lcSSc})$. Serum samples were also obtained from 5 systemic lupus erythematosus patients, 5 dermatomyositis patients, and 20 healthy subjects. Realtime PCR revealed that miR-150 levels in the sera of SSc patients were significantly lower than those in healthy subjects (Figure 4A). When SSc patients were considered separately by classification, both dcSSc patients and lcSSc patients showed decreased miR-150 concentration. The values in dermatomyositis patients and systemic lupus erythematosus patients were slightly decreased compared with those in healthy controls, but there were no significant differences.

Next, to determine whether serum miR-150 levels contribute to the pathogenesis of SSc, we analyzed the association between miR-150 levels and various clinical or laboratory features (Table 2). Although the difference did not reach statistical significance, patients with lower miR-150 levels had a higher ratio of dcSSc to $\mathrm{lcSSc}$ (15:12 versus 5:8) and a higher Modified Rodnan Skin Score (MRSS; 14.0 versus 8.3), compared with patients with normal levels of miR-150. A significantly higher incidence of antitopoisomerase I antibody and a higher prevalence of pitting scars were seen in patients with lower miR-150 levels than in those without $(58.3 \%$ versus $0 \%$ and $58.3 \%$ versus $12.5 \%$, respectively; $P<0.05$ ). Thus, our results suggest that patients with low miR-150 levels may exhibit more severe clinical manifestations.

\section{Discussion}

Recently, Maurer et $\mathrm{al}^{58}$ reported that down-regulation of miR-29a contributes to overexpression of extracellular matrix in SSc fibroblasts in vitro. Our present findings support the idea that miRNAs are involved in the pathogenesis of SSc. In addition, with this study we have demonstrated three major new findings.

First, we identified several miRNAs that were overexpressed or suppressed specifically in SSc fibroblasts, compared with normal fibroblasts, by miRNA PCR array. Among the down-regulated miRNAs, we focused on miR150 as the regulator of integrin $\beta 3$. Although the expression of miR-150 has been evaluated in gastric cancer and in chronic lymphocytic leukemia, ${ }^{59,60}$ the present study is the first to demonstrate the expression of miR-150 in rheumatic diseases (of which SSc is one).

Second, in the present study we also found new miRNAtarget interactions in dermal fibroblasts. Down-regulated
miR-150 leads to overexpression of integrin $\beta 3$ in normal fibroblasts, and overexpression of the miRNA leads to down-regulation of the integrin in SSc fibroblasts. Although integrin $\beta 3$ is thought to be the upstream key event in the TGF- $\beta$ activation process of SSc, the mechanism of integrin overexpression in SSc is unknown. Our results suggest that the down-regulation of miR-150, due to DNA methylation, can cause the overexpression of integrin directly, and can activate TGF- $\beta$ signaling without changing TGF- $\beta 1$ levels in SSc fibroblasts. Activated TGF- $\beta$ signaling induces phosphorylation of Smad3, which stimulates the transcriptional activation of collagen gene and the tissue fibrosis seen in SSc. ${ }^{10}$

Lastly, we investigated tissue and serum miRNA levels in SSc. To our knowledge, the present study is the first to show that miR-150 is detectable and quantifiable in serum. The miR-150 levels in serum from lcSSc and dcSSc patients were significantly lower than those in serum from healthy subjects. Also, SSc patients with lower serum miR-150 levels tended to have more severe clinical manifestation. Thus, serum miR-150 levels can be a disease marker. The diagnosis of SSc presents little problem when the clinical features have fully developed. It may be difficult to distinguish lcSSc from healthy skin, however, because skin sclerosis is sometimes not apparent in lcSSc, especially in very early stages. ${ }^{61}$ Serum levels of miR-150 may be useful for the differentiation of lcSSc from healthy skin. Serial time-course measurement of serum miR-150 concentration in suspect cases could lead to early detection of developing SSc.

Taken together, our results yield a hypothetical model of an miR-150-mediated SSc-phenotype (Figure 4B). The down-regulation of miR-150 may be the most upstream event in the TGF- $\beta$ activation process of SSc. Clarifying the involvement of miRNAs in the pathogenesis of SSc could lead to development of new diagnostic tools and new treatments using miRNA (eg, by transfection into the fibrotic lesion).

\section{Acknowledgments}

We thank Junko Suzuki, Chiemi Shiotsu, and Faith Chengetayi Muchemwa for their valuable technical assistance.

\section{Supplemental Data}

Supplemental material for this article can be found at http:// dx.doi.org/10.1016/j.ajpath.2012.09.023.

\section{References}

1. Korn JH: Immunologic aspects of scleroderma. Curr Opin Rheumatol 1989, 1:479-484

2. Mauch C, Kreig T: Fibroblast-matrix interactions and their role in the pathogenesis of fibrosis. Rheum Dis Clin North Am 1990, 16 : 93-107 
3. Mauch C, Kozlowska E, Eckes B, Krieg T: Altered regulation of collagen metabolism in scleroderma fibroblasts grown within threedimensional collagen gels. Exp Dermatol 1992, 1:185-190

4. Jelaska A, Arakawa M, Broketa G, Korn JH: Heterogeneity of collagen synthesis in normal and systemic sclerosis skin fibroblasts. Increased proportion of high collagen-producing cells in systemic sclerosis fibroblasts. Arthritis Rheum 1996, 39:1338-1346

5. Massagué J: The transforming growth factor-beta family. Annu Rev Cell Biol 1990, 6:597-641

6. LeRoy EC, Smith EA, Kahaleh MB, Trojanowska M, Silver RM: A strategy for determining the pathogenesis of systemic sclerosis. Is transforming growth factor beta the answer? Arthritis Rheum 1989, 32: 817-825

7. LeRoy EC: Increased collagen synthesis by scleroderma skin fibroblasts in vitro: a possible defect in the regulation or activation of the scleroderma fibroblast. J Clin Invest 1974, 54:880-889

8. Jimenez SA, Feldman G, Bashey RI, Bienkowski R, Rosenbloom J: Co-ordinate increase in the expression of type I and type III collagen genes in progressive systemic sclerosis fibroblasts. Biochem J 1986, 237:837-843

9. Kikuchi K, Hartl CW, Smith EA, LeRoy EC, Trojanowska M: Direct demonstration of transcriptional activation of collagen gene expression in systemic sclerosis fibroblasts: insensitivity to TGF beta 1 stimulation. Biochem Biophys Res Commun 1992, 187:45-50

10. Asano $\mathrm{Y}$, Ihn H, Yamane K, Kubo M, Tamaki K: Impaired Smad7-Smurf-mediated negative regulation of TGF-beta signaling in scleroderma fibroblasts. J Clin Invest 2004, 113:253-264

11. Ihn H, Yamane K, Kubo M, Tamaki K: Blockade of endogenous transforming growth factor beta signaling prevents up-regulated collagen synthesis in scleroderma fibroblasts: association with increased expression of transforming growth factor beta receptors. Arthritis Rheum 2001, 44:474-480

12. Lyons RM, Gentry LE, Purchio AF, Moses HL: Mechanism of activation of latent recombinant transforming growth factor beta 1 by plasmin. J Cell Biol 1990, 110:1361-1367

13. Ihn H: The role of TGF-beta signaling in the pathogenesis of fibrosis in scleroderma. Arch Immunol Ther Exp (Warsz) 2002, 50:325-331

14. Asano $\mathrm{Y}$, Ihn H, Yamane K, Jinnin M, Tamaki K: Increased expression of integrin alphavbeta5 induces the myofibroblastic differentiation of dermal fibroblasts. Am J Pathol 2006, 168:499-510

15. Schultz-Cherry S, Murphy-Ullrich JE: Thrombospondin causes activation of latent transforming growth factor-beta secreted by endothelial cells by a novel mechanism [Erratum appeared in J Cell Biol 1993, 122:following 1143]. J Cell Biol 1993, 122:923-932

16. Asano $\mathrm{Y}$, Ihn H, Jinnin M, Mimura $\mathrm{Y}$, Tamaki K: Involvement of alphavbeta5 integrin in the establishment of autocrine TGF-beta signaling in dermal fibroblasts derived from localized scleroderma. J Invest Dermatol 2006, 126:1761-1769

17. Asano Y, Ihn H, Yamane K, Jinnin M, Mimura Y, Tamaki K: Involvement of alphavbeta5 integrin-mediated activation of latent transforming growth factor betal in autocrine transforming growth factor beta signaling in systemic sclerosis fibroblasts. Arthritis Rheum 2005, 52:2897-2905

18. Asano $\mathrm{Y}$, Ihn H, Yamane K, Jinnin M, Mimura Y, Tamaki K: Increased expression of integrin alpha(v)beta3 contributes to the establishment of autocrine TGF-beta signaling in scleroderma fibroblasts. J Immunol 2005, 175:7708-7718

19. Bartel DP: MicroRNAs: genomics, biogenesis, mechanism, and function. Cell 2004, 116:281-297

20. Denli AM, Tops BB, Plasterk RH, Ketting RF, Hannon GJ: Processing of primary microRNAs by the Microprocessor complex. Nature 2004, 432:231-235

21. Herrera BM, Lockstone HE, Taylor JM, Ria M, Barrett A, Collins S, Kaisaki P, Argoud K, Fernandez C, Travers ME, Grew JP, Randall JC, Gloyn AL, Gauguier D, McCarthy MI, Lindgren CM: Global microRNA expression profiles in insulin target tissues in a spontaneous rat model of type 2 diabetes. Diabetologia 2010, 53:1099-1109
22. Kuehbacher A, Urbich C, Dimmeler S: Targeting microRNA expression to regulate angiogenesis. Trends Pharmacol Sci 2008, 29: $12-15$

23. Furer V, Greenberg JD, Attur M, Abramson SB, Pillinger MH: The role of microRNA in rheumatoid arthritis and other autoimmune diseases. Clin Immunol 2010, 136:1-15

24. Davidson-Moncada J, Papavasiliou FN, Tam W: MicroRNAs of the immune system: roles in inflammation and cancer. Ann N Y Acad Sci 2010, 1183:183-194

25. Ihn H, LeRoy EC, Trojanowska M: Oncostatin M stimulates transcription of the human alpha2(I) collagen gene via the Sp1/Sp3binding site. J Biol Chem 1997, 272:24666-24672

26. Ihn H, Sato S, Fujimoto M, Kikuchi K, Igarashi A, Soma Y, Tamaki K, Takehara K: Measurement of anticardiolipin antibodies by ELISA using beta 2-glycoprotein I (beta 2-GPI) in systemic sclerosis. Clin Exp Immunol 1996, 105:475-479

27. LeRoy EC, Black C, Fleischmajer R, Jablonska S, Krieg T, Medsger TA Jr, Rowell N, Wollheim F: Scleroderma (systemic sclerosis): classification, subsets and pathogenesis. J Rheumatol 1988, 15: 202-205

28. Matsushita T, Fujimoto M, Hasegawa M, Matsushita Y, Komura K, Ogawa F, Watanabe R, Takehara K, Sato S: BAFF antagonist attenuates the development of skin fibrosis in tight-skin mice. J Invest Dermatol 2007, 127:2772-2780

29. Martin MM, Buckenberger JA, Jiang J, Malana GE, Nuovo GJ, Chotani M, Feldman DS, Schmittgen TD, Elton TS: The human angiotensin II type 1 receptor $+1166 \mathrm{~A} / \mathrm{C}$ polymorphism attenuates microRNA-155 binding. J Biol Chem 2007, 282:24262-24269

30. Nuovo GJ: In situ detection of precursor and mature microRNAs in paraffin embedded, formalin fixed tissues and cell preparations. Methods 2008, 44:39-46

31. Nakashima T, Jinnin M, Etoh T, Fukushima S, Masuguchi S, Maruo K, Inoue $\mathrm{Y}$, Ishihara T, Ihn H: Down-regulation of mir-424 contributes to the abnormal angiogenesis via MEK1 and cyclin E1 in senile hemangioma: its implications to therapy. PLoS One 2010, 5:e14334

32. Makino T, Jinnin M, Muchemwa FC, Fukushima S, KogushiNishi H, Moriya C, Igata T, Fujisawa A, Johno T, Ihn H: Basic fibroblast growth factor stimulates the proliferation of human dermal fibroblasts via the ERK1/2 and JNK pathways. Br J Dermatol 2010, 162:717-723

33. Kroh EM, Parkin RK, Mitchell PS, Tewari M: Analysis of circulating microRNA biomarkers in plasma and serum using quantitative reverse transcription-PCR (qRT-PCR) [Erratum appeared in Methods 2010, 52:268]. Methods 2010, 50:298-301

34. Griffiths-Jones S: The microRNA Registry. Nucleic Acids Res 2004, 32(Database issue):D109-D111

35. Griffiths-Jones S, Grocock RJ, van Dongen S, Bateman A, Enright AJ: miRBase: microRNA sequences, targets and gene nomenclature. Nucleic Acids Res 2006, 34(Database issue):D140-D144

36. Griffiths-Jones S, Saini HK, van Dongen S, Enright AJ: miRBase: tools for microRNA genomics. Nucleic Acids Res 2008, 36(Database issue):D154-D158

37. Kozomara A, Griffiths-Jones S: miRBase: integrating microRNA annotation and deep-sequencing data. Nucleic Acids Res 2011, 39(Database issue):D152-D157

38. Yamane K, Suzuki H, Ihn H, Kato M, Yoshikawa H, Tamaki K: Cell type-specific regulation of the TGF-beta-responsive alpha2(I) collagen gene by CpG methylation. J Cell Physiol 2005, 202: $822-830$

39. Lewis BP, Burge CB, Bartel DP: Conserved seed pairing, often flanked by adenosines, indicates that thousands of human genes are microRNA targets. Cell 2005, 120:15-20

40. Friedman RC, Farh KK, Burge CB, Bartel DP: Most mammalian mRNAs are conserved targets of microRNAs. Genome Res 2009, 19:92-105

41. Grimson A, Farh KK, Johnston WK, Garrett-Engele P, Lim LP, Bartel DP: MicroRNA targeting specificity in mammals: determinants beyond seed pairing. Mol Cell 2007, 27:91-105 
42. Garcia DM, Baek D, Shin C, Bell GW, Grimson A, Bartel DP: Weak seed-pairing stability and high target-site abundance decrease the proficiency of 1sy-6 and other microRNAs. Nat Struct Mol Biol 2011, 18:1139-1146

43. Betel D, Koppal A, Agius P, Sander C, Leslie C: Comprehensive modeling of microRNA targets predicts functional non-conserved and non-canonical sites. Genome Biol 2010, 11:R90

44. Betel D, Wilson M, Gabow A, Marks DS, Sander C: The microRNA.org resource: targets and expression. Nucleic Acids Res 2008, 36(Database issue):D149-D153

45. John B, Enright AJ, Aravin A, Tuschl T, Sander C, Marks DS: Human microRNA targets [Erratum appeared in PLoS Biol 2005, 3:e264]. PLoS Biol 2004, 2:e363

46. Enright AJ, John B, Gaul U, Tuschl T, Sander C, Marks DS: MicroRNA targets in Drosophila. Genome Biol 2003, 5:R1

47. Maragkakis M, Alexiou P, Papadopoulos GL, Reczko M, Dalamagas T, Giannopoulos G, Goumas G, Koukis E, Kourtis K, Simossis VA, Sethupathy P, Vergoulis T, Koziris N, Sellis T, Tsanakas P, Hatzigeorgiou AG: Accurate microRNA target prediction correlates with protein repression levels. BMC Bioinformatics 2009, 10:295

48. Maragkakis M, Reczko M, Simossis VA, Alexiou P, Papadopoulos GL, Dalamagas T, Giannopoulos G, Goumas G, Koukis E, Kourtis K, Vergoulis T, Koziris N, Sellis T, Tsanakas P, Hatzigeorgiou AG: DIANA-microT web server: elucidating microRNA functions through target prediction. Nucleic Acids Res 2009, 37(Web Server issue):W273-W276

49. Wang $X$, El Naqa IM: Prediction of both conserved and nonconserved microRNA targets in animals. Bioinformatics 2008, 24: 325-332

50. Wang $\mathrm{X}$ : miRDB: a microRNA target prediction and functional annotation database with a wiki interface. RNA 2008, 14:1012-1017

51. Long JM, Lahiri DK: MicroRNA-101 downregulates Alzheimer's amyloid-beta precursor protein levels in human cell cultures and is differentially expressed. Biochem Biophys Res Commun 2011, 404: 889-895

52. Suzuki H, Yamamoto E, Nojima M, Kai M, Yamano HO, Yoshikawa K, Kimura T, Kudo T, Harada E, Sugai T, Takamaru H, Niinuma T, Maruyama R, Yamamoto H, Tokino T, Imai K, Toyota M, Shinomura Y: Methylation-associated silencing of microRNA-34b/c in gastric cancer and its involvement in an epigenetic field defect. Carcinogenesis 2010, 31:2066-2073
53. Gilad S, Meiri E, Yogev Y, Benjamin S, Lebanony D, Yerushalmi N, Benjamin H, Kushnir M, Cholakh H, Melamed N, Bentwich Z, Hod M, Goren Y, Chajut A: Serum microRNAs are promising novel biomarkers. PLoS One 2008, 3:e3148

54. Chen X, Ba Y, Ma L, Cai X, Yin Y, Wang K, Guo J, Zhang Y, Chen J, Guo X, Li Q, Li X, Wang W, Wang J, Jiang X, Xiang Y, Xu C, Zheng P, Zhang J, Li R, Zhang H, Shang X, Gong T, Ning G, Zen K, Zhang CY: Characterization of microRNAs in serum: a novel class of biomarkers for diagnosis of cancer and other diseases. Cell Res 2008, 18:997-1006

55. Mitchell PS, Parkin RK, Kroh EM, Fritz BR, Wyman SK, PogosovaAgadjanyan EL, Peterson A, Noteboom J, O'Briant KC, Allen A, Lin DW, Urban N, Drescher CW, Knudsen BS, Stirewalt DL, Gentleman R, Vessella RL, Nelson PS, Martin DB, Tewari M: Circulating microRNAs as stable blood-based markers for cancer detection. Proc Natl Acad Sci USA 2008, 105:10513-10518

56. Hunter MP, Ismail N, Zhang X, Aguda BD, Lee EJ, Yu L, Xiao T, Schafer J, Lee ML, Schmittgen TD, Nana-Sinkam SP, Jarjoura D, Marsh CB: Detection of microRNA expression in human peripheral blood microvesicles [Erratum appeared in PLoS One 2010, 5(3) doi: 10.1371/annotation/b15ca816-7b62-4474-a568-6b60b8959742]. PLoS One 2008, 3:e3694

57. Valenti R, Huber V, Iero M, Filipazzi P, Parmiani G, Rivoltini L: Tumor-released microvesicles as vehicles of immunosuppression. Cancer Res 2007, 67:2912-2915

58. Maurer B, Stanczyk J, Jüngel A, Akhmetshina A, Trenkmann M, Brock M, Kowal-Bielecka O, Gay RE, Michel BA, Distler JH, Gay S, Distler O: MicroRNA-29, a key regulator of collagen expression in systemic sclerosis. Arthritis Rheum 2010, 62:1733-1743

59. Wu Q, Jin H, Yang Z, Luo G, Lu Y, Li K, Ren G, Su T, Pan Y, Feng B, Xue Z, Wang X, Fan D: miR-150 promotes gastric cancer proliferation by negatively regulating the pro-apoptotic gene EGR2. Biochem Biophys Res Commun 2010, 392:340-345

60. Wang M, Tan LP, Dijkstra MK, van Lom K, Robertus JL, Harms G, Blokzijl T, Kooistra K, van T'veer MB, Rosati S, Visser L, JongenLavrencic M, Kluin PM, van den Berg A: miRNA analysis in B-cell chronic lymphocytic leukaemia: proliferation centres characterized by low miR-150 and high BIC/miR-155 expression. J Pathol 2008, 215: $13-20$

61. Ihn H, Sato S, Tamaki T, Soma Y, Tsuchida T, Ishibashi $\mathrm{Y}$, Takehara K: Clinical evaluation of scleroderma spectrum disorders using a points system. Arch Dermatol Res 1992, 284:391-395 